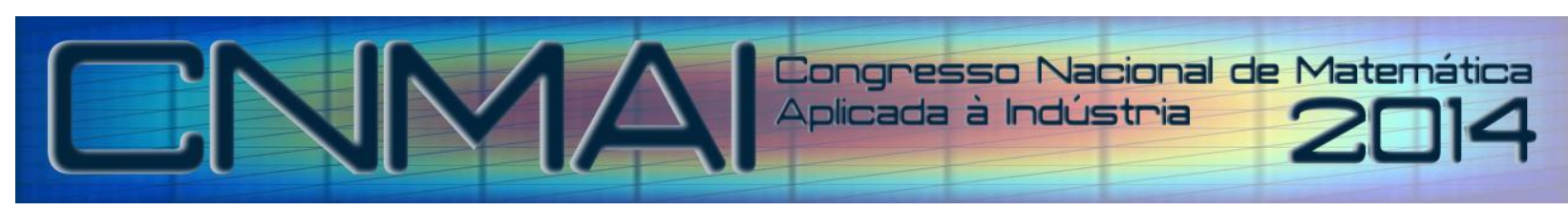

18 a 21 de novembro de 2014, Caldas Novas - Goiás

\title{
ESTIMATIVA DOS EFEITOS DA APROXIMAÇÃO DAS INCLUSÕES NAS CONDIÇÕES DE CONTORNO DE UM MATERIAL BIFÁSICO
}

\author{
Camila Francisca de Melo, mila_f_melo@hotmail.com ${ }^{1}$ \\ Marco André Argenta, marco.argenta@gmail.com² \\ ${ }^{1}$ Universidade Federal do Paraná - Programa de Pós-Graduação em Métodos Numéricos em Engenharia, Centro \\ Politécnico da UFPR - Curitiba - Paraná/Brasil \\ ${ }^{2}$ Universidade Federal do Paraná - Programa de Pós-Graduação em Métodos Numéricos em Engenharia, Centro \\ Politécnico da UFPR - Curitiba - Paraná/Brasil
}

\begin{abstract}
Resumo: Considerando um material bifásico isotrópico (inclusão e matriz), o intuito deste trabalho é analisar o comportamento e a geometria de inclusões em um corpo. Esta estimativa se fará a partir da localização da inclusão na matriz, iniciando com uma inclusão centralizada e aproximando-a da condição de contorno. Esta hipótese será testada entre uma estrutura homogeneizada e outra com inclusão e matriz explícitas. Para que isso ocorra, faz-se o uso do método de homogeneização de Mori-Tanaka, afim de se obter o tensor constitutivo efetivo para um sólido bifásico isotrópico. Para o cálculo das propriedades efetivas do compósito foi implementado um algoritmo em linguagem Python, enquanto que para a modelagem e discretização do material em elementos finitos utiliza-se o software Ansys. A avaliação deste artigo se resume em uma análise comparativa dos resultados obtidos, que se mostraram eficaz, além de proporcionar uma reflexão do efeito das inclusões nas condições de contorno da matriz.
\end{abstract}

Palavras-chave: métodos numéricos, método de homogeneização, inclusão, material bifásico, elementos finitos.

\section{INTRODUÇÃO}

O problema da inclusão possui grande importância no campo da micromecânica, pois, em conjunto com outras técnicas, consegue representar os campos elásticos de compósitos bifásicos heterogêneos com várias inclusões. Eshelby (1957) discute um método que tem por finalidade encontrar o campo elástico de uma inclusão elipsoidal em um sólido elástico quando uma região inclusa, denominada inclusão, sofre uma variação de forma e tamanho, designada deformação homogênea uniforme (Eshelby, 1957). Desde então a micromecânica vem gerando gradativamente um maior interesse para o problema da inclusão.

Dentre os trabalhos encontrados neste conceito, podem ser citados: o livro clássico de Mura (1987), o trabalho de Eshelby (1957) sobre inclusões elipsoidais, Xu \& Wang (2005) abordando inclusões poligonais, Tian \& Rajapakse (2007) e os estudos sobre uma heterogeneidade elíptica em nanoescala, Zou et al (2010) e as inclusões não elípticas, Lorenci (2013) trabalhando com o comportamento poroelástico de um meio rochoso fraturado.

Neste trabalho, será dada ênfase a um compósito bifásico isotrópico com uma perturbação constante, de modo a apresentar a solução de uma matriz retangular com uma inclusão imersa no formato circular de diferentes materiais. Para isso empregou-se a técnica de homogeneização e de um método numérico, denominado método dos elementos finitos utilizado na modelagem do problema.

O artigo está disposto da seguinte modo: na seção 2, o problema da inclusão é apresentado exibindo as equações e definindo-as para o material isotrópico; já na seção 3 é descrita uma breve introdução sobre o método de homogeneização de Mori-Tanaka que será utilizado no desenvolvimento deste trabalho. A seção 4 traz os resultados computacionais obtidos na avaliação das estruturas. Para finalizar, na seção 5 são mostradas as considerações finais do artigo.

\section{DESCRIÇÃO E O PROBLEMA DA INCLUSÃO}

Segundo Rodin (1996), o estudo sobre inclusão é relevante por duas razões principais: a primeira está relacionada com o fato de o modelo poder ser utilizado na representação de muitos fenômenos que envolvem a expansão térmica e 
transformações estruturais nos sólidos, enquanto a segunda está relacionada com o problema de heterogeneidade em uma matriz infinita.

O problema da inclusão abordado neste trabalho considera um sólido linear bifásico homogêneo de domínio $D$, com um subdomínio $\Omega$, denominado respectivamente matriz e inclusão, como representado na Fig. 1. Perturbações no campo de deformação ${ }^{1}$, tais como expansão térmica, transformação de fase, deformação inicial e deformação plástica, designadas $\varepsilon_{i j}^{*}(x)$ em um subdomínio $\Omega$ que assume valor zero no domínio remanescente $D-\Omega$. As perturbações no campo de deformações $\varepsilon_{i j}^{*}(x)$ serão assumidas como constantes, a exemplo de Mura (1987).

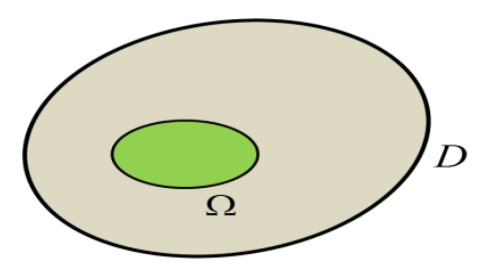

\section{Figura 1. Sólido linear se domínio $D$ e subdomínio $\Omega$.}

O modelo proposto neste trabalho segue as condições acima definidas, considerando uma circunferência de aço inclusa em uma chapa retangular, denominadas respectivamente, inclusão circular e matriz como podem ser visto na Fig. 2.

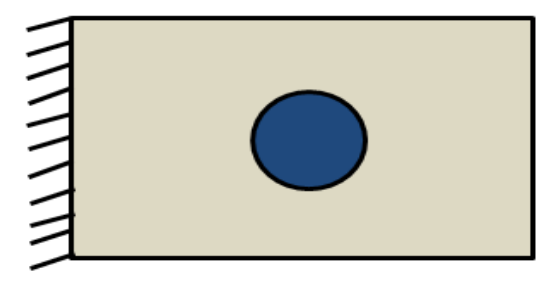

Figura 2. Inclusão circular imersa em uma placa retangular (matriz).

\subsection{Inclusão}

Utilizando a relação deformação e deslocamento, é possível obter expressões explícitas para o campo de tensão e deformação tanto dentro como fora da inclusão, que no caso foram escritas em notação indicial. De acordo com Weinberger, Cai \& Barnett (2005) e Eshelby (1957), o campo de deformações totais $\varepsilon_{i j}$ pode ser expresso da seguinte maneira:

$$
\varepsilon_{i j}=\frac{1}{2}\left(\frac{\partial u_{i}}{\partial x_{j}}+\frac{\partial u_{j}}{\partial x_{i}}\right)
$$

onde que $\partial u_{i} / \partial x_{j}$ é a derivada parcial do deslocamento $u_{i}$ com relação a direção $j$ da variável $x$

Para pequenas deformações consideradas no decorrer deste trabalho, a deformação total $\varepsilon_{i j}$ é escrita como a soma da deformação elástica $\epsilon_{i j}$ e as perturbações no campo de deformações $\varepsilon_{i j}^{*}(x)$.

$$
\varepsilon_{i j}=\epsilon_{i j}+\varepsilon_{i j}^{*}
$$

A relação entre tensão $\sigma_{i j}$ e deformação segundo Mura (1987), pode ser expressa com o auxilio da Lei de Hooke, da seguinte maneira:

$$
\begin{gathered}
\sigma_{i j}=C_{i j k l} \epsilon_{k l}=C_{i j k l}\left(\varepsilon_{k l}-\varepsilon_{k l}^{*}\right) \\
\sigma_{i j}=C_{i j k l}\left(u_{k, l}-\varepsilon_{k l}^{*}\right)
\end{gathered}
$$

sendo a tensão e a deformação são tensores de segunda ordem, enquanto $C_{i j k l}$ é um tensor de quarta ordem conhecido como tensor constitutivo de rigidez ( Azevedo, 2007).

${ }^{1}$ Perturbações no campo de deformações podem ser encontradas na literatura como eigenstrain ou deformações inelásticas (Argenta, 2011, apud Mura, 1987). 
Considerando as condições de equilíbrio $\sigma_{i j, j}=0$, tem-se que:

$$
C_{i j k l} u_{k, l j}=C_{i j k l} \varepsilon_{k l, j}^{*}
$$

A perturbação no campo de deformação gera um deslocamento na matriz, que segundo Mura (1987) pode ser considerado como um deslocamento $u_{i}$ causado por uma força de corpo $-C_{i j k l} \varepsilon_{k l, j}^{*}$ aplicado na direção de $x_{i}$ do ponto $x^{\prime}$. Se $G_{i j}\left(x-x^{\prime}\right)$ é a solução por unidade de força aplicada na direção de $x_{j}$, segundo Kawashita \& Nozaki (2001) escreve-se:

$$
G_{i j}\left(x-x^{\prime}\right)=\frac{1}{8 \pi(1-v) \mu}\left[-(3-4 v) \delta_{i j} \log R+\frac{\left(x_{i}-x_{i}^{\prime}\right)\left(x_{j}-x_{j}^{\prime}\right)}{R^{2}}\right]
$$

onde $R^{2}=\left(x_{i}-x_{i}^{\prime}\right)^{2}+\left(x_{j}-x_{j}^{\prime}\right)^{2}$, na expressão acima, $\mu$ e $v$ correspondem respectivamente módulo de cisalhamento e o coeficiente de Poisson, enquanto $\delta_{i j}$ é o delta de Kronecker. Por fim, $G_{i j}\left(x-x^{\prime}\right)$ é conhecido como a função de Green bidimensional para o estado plano de deformação de um material isotrópico.

Com base nas expressões anteriores, Eq. (2) a Eq. (5) é possível determinar os campos elásticos de deslocamento e deformação nas diferentes posições da inclusão. Em (Mura, 1987) esses campos de deslocamentos são expressos por:

$$
\begin{gathered}
u_{i}(x)=-C_{j k m n} \varepsilon_{m n}^{*}\left(x^{\prime}\right) \int_{\Omega} G_{i j, k}\left(x-x^{\prime}\right) d x^{\prime} \\
\varepsilon_{i j}(x)=-\frac{1}{2} C_{k l m n} \varepsilon_{m n}^{*}\left(x^{\prime}\right) \int_{\Omega}\left(G_{i k, l j}\left(x-x^{\prime}\right)+G_{j k, l i}\left(x-x^{\prime}\right) d x^{\prime}\right)
\end{gathered}
$$

sendo que $G_{i j, k}\left(x-x^{\prime}\right)=\partial / \partial x_{k} G_{i j}\left(x-x^{\prime}\right)=-\partial / \partial x_{k}^{\prime} G_{i j}\left(x-x^{\prime}\right)$.

Como mencionado anteriormente $C_{j k m n}$ é um tensor constitutivo de rigidez de quarta ordem; de acordo com Xu \& Wang (2005), pode ser representado da seguinte maneira:

$$
C_{j k m n}=\lambda \delta_{j k} \delta_{m n}+\mu\left(\delta_{j m} \delta_{k n}+\delta_{j n} \delta_{k m}\right)
$$

onde $\lambda=2 \mu v /(1-2 v)$, com $\lambda$ e $\mu$ constantes de Lamé e $\delta_{i j}$ é o delta de Kronecker. É fácil observar que esse tensor depende apenas de duas variáveis, devido ao comportamento do material isotrópico.

\section{HOMOGENEIZAÇÃO}

A homogeneização é uma ferramenta matemática que tem como finalidade determinar as propriedades mecânicas equivalentes ou efetivas dos materiais heterogêneos (Soares, 2010). Este procedimento parte da especificação das fases que compõem o meio heterogêneo considerando as características geométricas e mecânicas de todos os constituintes e suas distribuições no compósito (Ma, 2010), (Lorenci, 2013).

A estimativa das propriedades mecânicas de materiais compósitos com frações volumétricas maiores do que uma pequena porcentagem ${ }^{2}$ (Cavalcante, 2006), leva em conta as interações entre as inclusões, ou seja, o efeito causado pelas heterogeneidades circundantes sobre os campos de deformação ou tensão (Böhm, 2010). Uma maneira de obter esses efeitos é aproximar estas tensões que atuam nas heterogeneidades, aplicadas longe do campo de deformações por uma matriz de deformação média. Combinando o conceito de matriz de tensão média com inclusão equivalente de Eshelby, tem-se a base do método proposto neste trabalho, o método de Mori-Tanaka para o cálculo das propriedades efetivas do compósito bifásico (Böhm, 2010).

\subsection{Método de Mori-Tanaka}

Segundo a abordagem apresentada por Benveniste (1987), o método de Mori-Tanaka considera um compósito de N fases e aproxima o efeito da interação entre as fases admitindo que a tensão em cada fase seja igual á tensão envolta na matriz submetida a um campo de deformação média (Almeida et. al., 2010). Com isso, o carregamento das heterogeneidades depende da existência das demais através da deformação da matriz (Lorenci, 2013).

A partir do conceito do método de homogeneização de Mori-Tanaka tem-se a seguinte equação (Tavares, Silva \& Silva, 2012):

$$
C=\left[f_{\alpha} C_{\alpha}\left[I-S C_{m}^{-1}\left(C_{m}-C_{\alpha}\right)\right]^{-1}+\left(1-f_{\alpha}\right) C_{m}\right]\left[f_{\alpha}\left[I-S C_{m}^{-1}\left(C_{m}-C_{\alpha}\right)\right]^{-1}+\left(1-f_{\alpha}\right) I\right]^{-1}
$$

\footnotetext{
${ }^{2}$ Cavalcante (2006) considera uma pequena porcentagem de $10 \%$.
} 
na expressão acima, $C_{m}$ é o tensor constitutivo da matriz, $C_{\alpha}$ é o tensor constitutivo da inclusão, $f_{\alpha}$ é a fração volumétrica da inclusão, $S$ é o tensor de Eshelby, $I$ é o tensor identidade e $C$ é o tensor constitutivo efetivo.

O tensor de quarto ordem $S$ é definido como uma transformação linear que relaciona perturbações no campo de deformação, função de forma e propriedades do material da inclusão e da matriz, com a deformação total, esse tensor será denominado ao longo deste trabalho como tensor de Eshelby (Argenta, 2011 apud Mura, 1987), (Lai, Rubin \& Krempl, 1993).

Considerando por exemplo, uma inclusão circular que é adotada neste trabalho de coordenadas $x_{1}$ e $x_{2}$ e condições de contorno $\left(\frac{x_{1}}{a_{1}}\right)^{2}+\left(\frac{x_{2}}{a_{2}}\right)^{2} \leq 1, a_{1}=a_{2}$ em um meio elástico isotrópico o tensor de Eshelby tem o seguinte formato (Weinberger, Cai \& Barnett, 2005):

$$
S_{i j m n}=\frac{4 v-1}{8(1-v)} \delta_{i j} \delta_{m n}+\frac{3-4 v}{8(1-v)}\left(\delta_{i n} \delta_{j m}+\delta_{j n} \delta_{i m}\right)
$$

sendo $S_{i j m n}$ o tensor de Eshelby no formato circular, $\delta_{i j}$ o delta de Kronecker.

Outras expressões para as múltiplas configurações do tensor podem ser encontradas em Mura (1987), Eshelby (1957), acima o mesmo foi apresentado em forma circular devido ao uso deste no presente artigo.

Uma atenção maior é dada na construção do tensor constitutivo efetivo, pois a manipulação toda é realizada com tensores de quarta ordem, sendo obrigatório o uso de operações tensoriais. Que segundo Mura (1987), particularmente para este caso onde os tensores de quarta ordem possuem simetria, é possível o uso das constantes de Voigt $C_{i j}$ que se relacionam com as componentes do tensor de rigidez $C_{i j k l}$, desde que $C_{i j}=C_{j i}$, com a finalidade de facilitar os cálculos.

\section{TESTES E EXPERIMENTOS}

As simulações computacionais foram realizadas considerando três posições distintas para as inclusões. Um algoritmo em linguagem Python foi desenvolvido e utilizado para o cálculo das propriedades efetivas do modelo. Contudo para a modelagem em Elementos Finitos é utilizado o software Ansys versão 14.0. As análises foram efetuadas em um computador com processador Intel $(R)$ Core $^{\mathrm{TM}} i 5-3337$ de $1.80 \mathrm{GHz}$ memória RAM de $6 \mathrm{~GB}$ e sistema operacional de 64 bits.

No entanto todas as simulações seguem um padrão de chapa retangular como matriz, medindo $200 \mathrm{~mm}$ de comprimento por $100 \mathrm{~mm}$ de largura com uma inclusão circular imersa na matriz de raio igual a $25 \mathrm{~mm}$. A chapa retangular é engastada na extremidade esquerda contendo um carregamento distribuído na extremidade oposta, sendo a matriz constituída de alumínio e a inclusão de aço, respectivamente com as seguintes constantes elásticas $E=6.9 \times$ $10^{10} \mathrm{~Pa}$ e $v=0.33 ; E=2.07 \times 10^{11} \mathrm{~Pa}$ e $v=0.3$.

Determinar o valor da perturbação no campo de deformação é uma das estimas mais importantes do método proposto, esta pode ser representada como funções polinomiais, trigonométricas, constantes, entre outros. No entanto, neste artigo as perturbações no campo de deformações são utilizadas como constantes adquiridas a partir de uma relação da área da inclusão com a matriz. Como nos testes faz-se o uso de um padrão de estrutura da matriz e inclusão, esta relação permanece a mesma.

A análise do trabalho é realizada em três posições diferentes para a localização da inclusão, denominadas Posição A para a inclusão centralizada, Posição B para a inclusão fora do centro e a Posição C para a inclusão próxima da condição de contorno (engaste), como representado respectivamente pelas figuras Fig. 3, Fig. 4 e Fig. 5, vale ressaltar que o intuito deste é avaliar a aproximação da inclusão na condição de contorno.

Na Figura 3 é ilustrado a primeira localização, Posição A, considerando uma chapa retangular com inclusão centralizada na matriz:

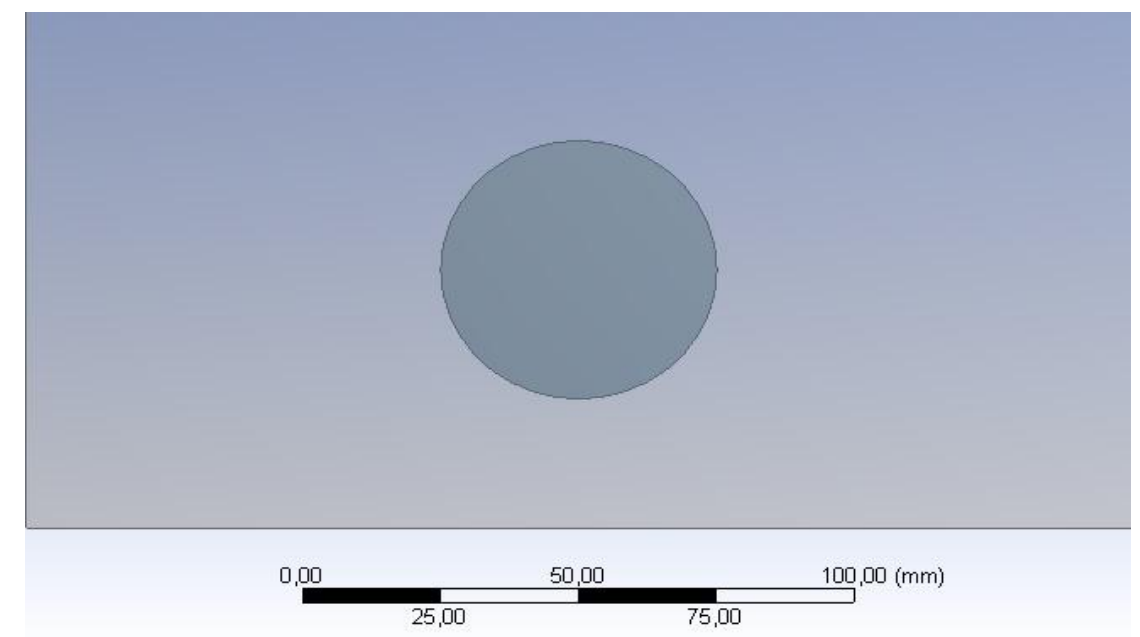


Figura 3. Posição A: Ilustração da modelagem da matriz retangular com uma inclusão centralizada.

Já na Figura 4 está representada a segunda posição da inclusão, onde analisa-se uma inclusão circular imersa em uma chapa retangular com as mesmas características definidas acima, porém distante do centro da matriz, ou seja, houve uma aproximação da inclusão a condição de contorno da chapa.

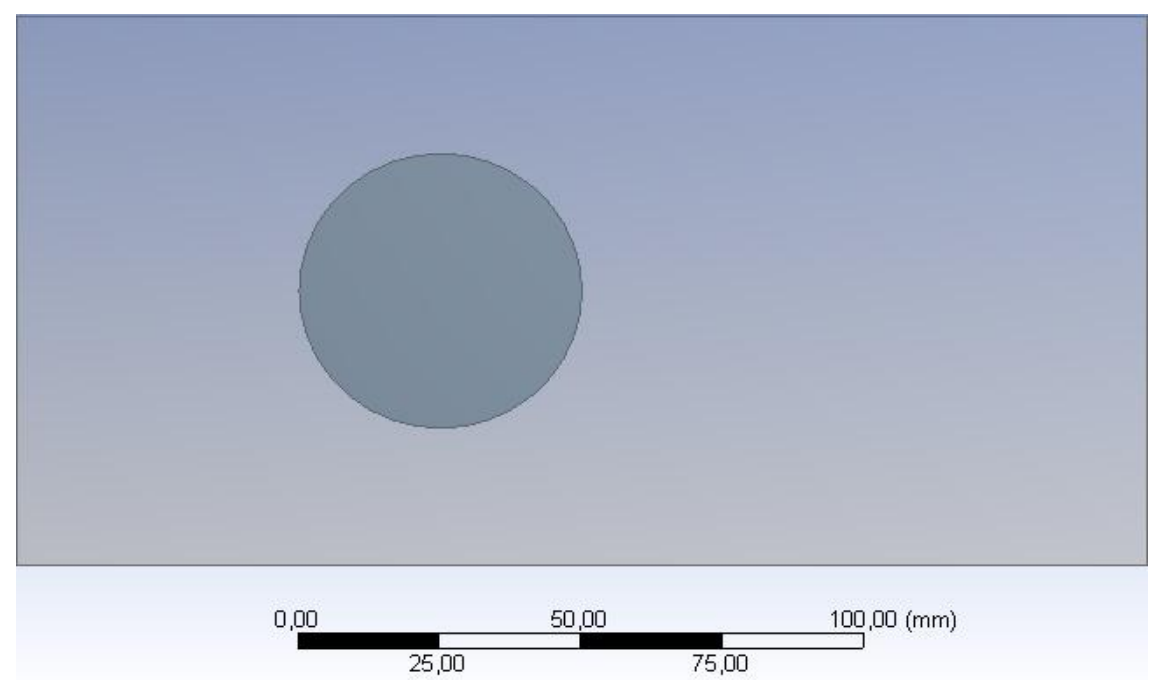

Figura 4. Posição B: Ilustração da modelagem da matriz retangular com uma inclusão fora do centro

Por sua vez na terceira disposição, a Posição C considera uma chapa retangular com a inclusão circular e as mesmas características das outras duas, mas agora a inclusão está bem próxima da aresta engastada da matriz. Como pode ser observado na Fig. 5.

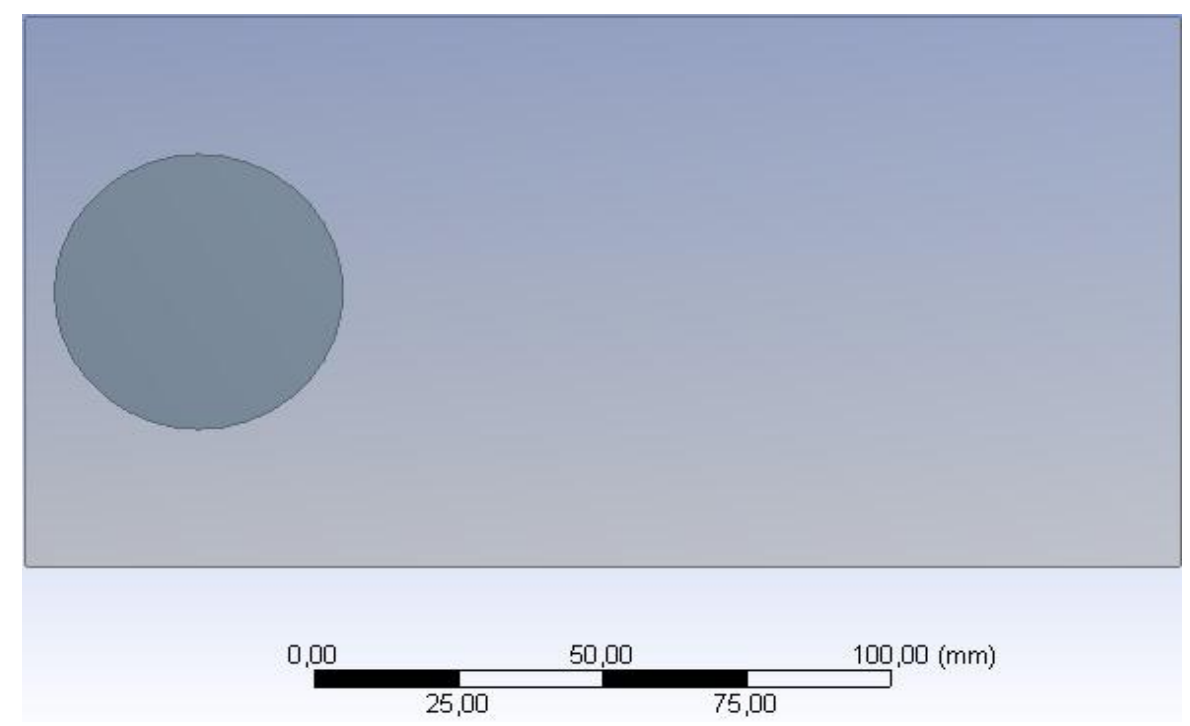

Figura 5. Posição C: Ilustração da modelagem da matriz retangular com uma inclusão próxima da aresta engastada.

Um algoritmo em linguagem Python denominado Tensor Efetivo que utiliza as Eq. (9) e Eq. (10), dá início ao desenvolvimento do artigo, pois verifica-se que as constantes elásticas da matriz e inclusão são diferentes, fazendo-se necessário o incremento e o uso do tensor constitutivo efetivo que represente o material do compósito bifásico.

Após efetuar os cálculos do tensor constitutivo efetivo, é implementado no mesmo algoritmo as Eq. (5) a Eq. (8) para encontrar os campos de deformação e deslocamento na aresta carregada da matriz. A variação da posição na inclusão, que a princípio está centralizada e é movida gradativamente na direção do apoio engastado da chapa são modeladas através dos limites de integração das Eq. (6) e Eq. (7); com isso os campos desejáveis podem ser encontrados sem maiores dificuldades.

Para cumprir os objetivos do trabalho, foram realizados testes computacionais com a modelagem de estruturas homogeneizadas (Tensor Efetivo), e assim comparadas com o software Ansys. Os deslocamentos totais e deformações 
encontradas utilizando perturbação no campo de deformações constantes, exibiram um comportamento bem próximo do encontrado no software Ansys que emprega o método dos Elementos Finitos.

Sendo assim, torna-se necessário expor a quantidade de nós e elementos utilizados na malha de elementos finitos, pois evidenciam a acurácia dos resultados obtidos. Com isso, na primeira posição foram usados um total de $3 \mathbf{5 9 9}$ elementos provenientes de uma quantidade de 11032 nós decorrentes da soma de 9028 nós da matriz e 2130 nós da inclusão, vale ressaltar que nesta adição têm-se a sobreposição dos nós na compatibilização entre as duas malhas geradas (matriz e inclusão). Já na Posição B gerou-se 2902 elementos na matriz e 772 elementos na inclusão, donde contabilizou um total de 11257 nós. Na última posição foi obtido um total de 11291 nós que originaram 3684 elementos. Deste modo, as malhas geradas resultou os bons resultados exibidos abaixo.

Os deslocamentos totais abaixo ilustrados, dizem respeito a borda engastada da matriz:

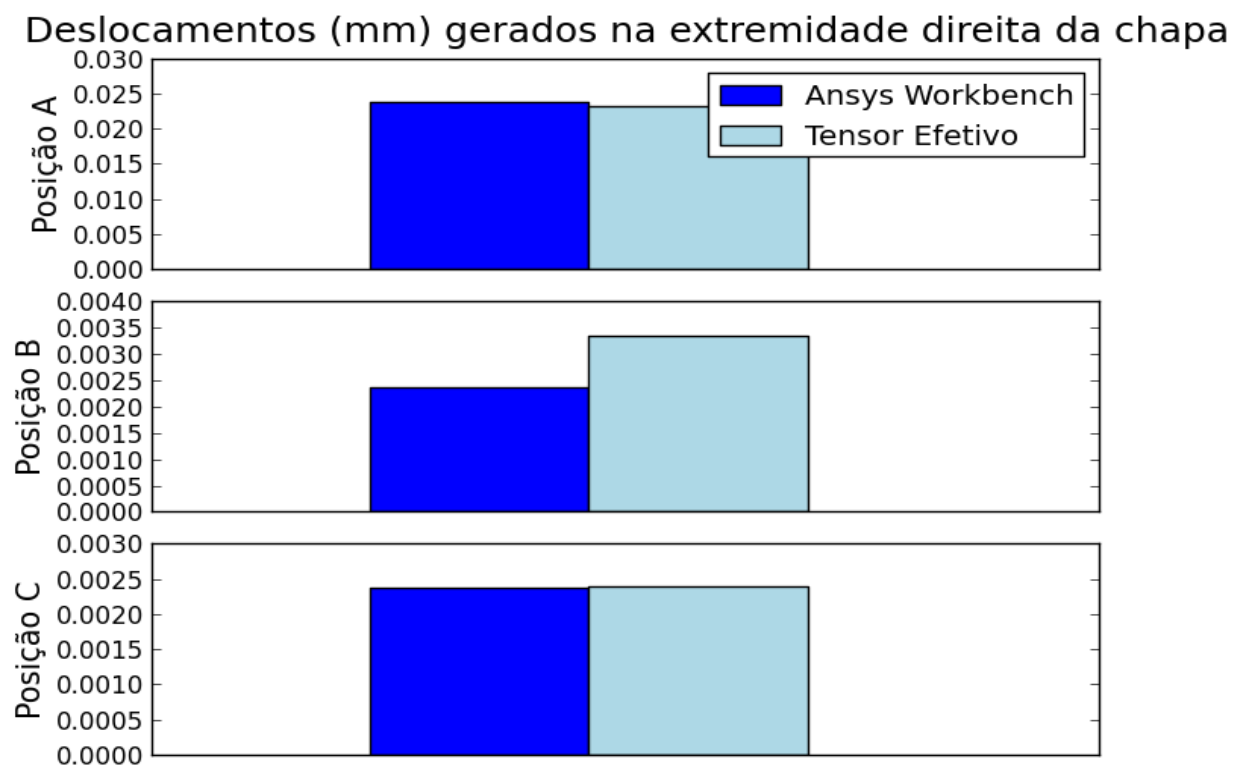

Figura 6. Gráficos comparativos dos deslocamentos totais das respectivas posições da inclusão.

Utilizando a Figura 6, pode-se notar que a comparação dos deslocamentos, quando a inclusão está localizada no centro da matriz (Posição A) e quando está mais próxima da aresta engastada (Posição C), tem-se os melhores resultados, com diferenças em módulo respectivamente de $6.246013 \cdot 10^{-4} \mathrm{~mm}$ e $3.400 \cdot 10^{-5} \mathrm{~mm}$. Contudo, já na Posição B é observado a maior alteração entre os dois métodos, chegando a uma diferença de $9.8914723 \cdot 10^{-4} \mathrm{~mm}$, como pode ser melhor visualizado na Tab.1.

Tabela 1. Resultados dos deslocamentos na extremidade com carregamento distribuído da chapa.

\begin{tabular}{l|c|c}
\hline & Deslocametos Ansys & Deslocamentos Tensor Efetivo \\
\hline Posição A & $2.3798 \cdot 10^{-2} \mathrm{~mm}$ & $2.31733987 \cdot 10^{-2} \mathrm{~mm}$ \\
\hline Posição B & $2.3633 \cdot 10^{-3} \mathrm{~mm}$ & $3.35244723 \cdot 10^{-3} \mathrm{~mm}$ \\
\hline Posição C & $2.3645 \cdot 10^{-3} \mathrm{~mm}$ & $2.3985 \cdot 10^{-3} \mathrm{~mm}$ \\
\hline
\end{tabular}

Outra análise interessante é o comportamento que o deslocamento tem a medida que a posição da inclusão se movimenta. Nota-se que a variação da Posição A para a Posição B gera a maior queda nos deslocamentos apresentados, pois observando a Posição B para a Posição C, este declínio continua porém menos acentuado.

A próxima figura ilustra a comparação da deformação da chapa entre o software Ansys e o Tensor Constitutivo Efetivo. 


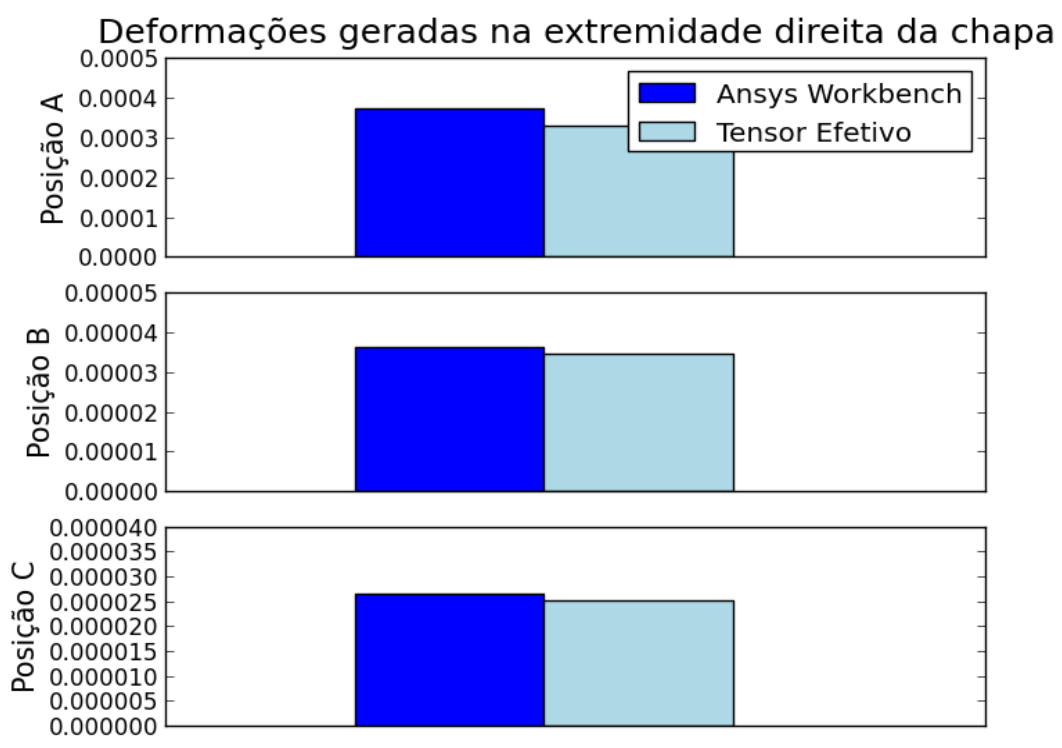

Figura 7. Gráficos comparativos das deformações totais das respectivas posições da inclusão.

As deformações totais referentes a extremidade cujo carregamento esta distribuído representadas na Fig. 7, mostraram-se mais próximas na Posição B e na Posição C, com uma alteração de aproximadamente $1.4680229 \cdot 10^{-6}$ na segunda localização da inclusão, e $1.4940 \cdot 10^{-6}$ na última localização. Já a Posição A, apresenata a maior diferença do teste com $4.332716 \cdot 10^{-5}$ de alteração entre os modelos propostos, como pode ser melhor visualizado na Tab. 2 . Contudo, pode-se afirmar que os resultados adquiridos são bons, pois ressalta-se que as perturbações no campo de deformações utilizadas são constantes.

Na Tabela 2 a seguir, estão explícitos os valores encontrados para as deformações totais utilizados na construção da Fig. 7:

Tabela 2. Resultados das deformações na extremidade com carregamento distribuído da chapa.

\begin{tabular}{l|c|c}
\hline & Deformações Ansys & Deformações Tensor Efetivo \\
\hline Posição A & $3.7408 \cdot 10^{-4}$ & $3.3075284 \cdot 10^{-4}$ \\
\hline Posição B & $3.6211 \cdot 10^{-5}$ & $3.47429771 \cdot 10^{-5}$ \\
\hline Posição C & $2.6653 \cdot 10^{-5}$ & $2.5159 \cdot 10^{-5}$ \\
\hline
\end{tabular}

Avaliando um pouco mais a Tab. 2 e a Fig. 7, verifica-se uma queda considerável de deformação entre a Posição A e a Posição B, no entanto entre a Posição B e a Posição C observa-se o mesmo comportamento, porém agora a deformação que ocorre é menor do que a encontrada entre as duas primeiras posições.

Com o auxílio da Tab. 1 e da Fig. 6 nota-se que quanto menor for a distância entre a inclusão e o engaste, o deslocamento tende a diminuir, contudo entre as posições que estão mais próximas da borda engastada é apresentado um deslocamento mínimo. Deste modo analisando as deformações que correspondem a Tab. 2 e a Fig. 7, percebe-se que assim como no deslocamento ocorre um declínio a medida que a inclusão é movida do centro do material, na deformação tem-se o mesmo comportamento, porém a alteração entre as duas últimas posições não são tão pequenas, quanto no deslocamento. Entretanto, confrontando os resultados obtidos nas deformações e nos deslocamentos das três posições, a inclusão mais afastada do engaste (posição centralizada) apresenta a maior deformação e deslocamento.

\section{COSIDERAÇÕES FINAIS}

A análise dos resultados encontrados revelam que os objetivos do trabalho foram alcançados, pois o método de MoriTanaka desenvolvido na linguagem de programação Python, se mostrou eficaz para a proposta do estudo de um compósito bifásico isotrópico, considerando uma região retangular com uma inclusão circular em diferentes posições.

Mesmo que estimado as perturbações no campo de deformações como constantes, as soluções obtidas com o Tensor Constitutivo Efetivo apresentou-se muito próximas dos resultados fornecidos pelo software Ansys, que utiliza o método dos elementos finitos. Sendo possível afirmar que quanto mais distante da extremidade engastada a inclusão esta, maior será a deformação e o deslocamento por ela adquirido, evidenciando também que o comportamento das três posições seguem um padrão de declínio, entretanto menos acentuado nos deslocamentos entre as duas últimas posições. 


\title{
REFERÊNCIAS
}

Almeida, F. P. A., Romão Filho, R. S., Cavalcante, R. W. B. \& Santos Júnior, A. 2010. Avaliação numérica do erro de modelagem global em estruturas constituídas por materiais heterogêneos. Proceedings of the IX Argentine Congress on Computational Mechanics and II South American Congress on Computational Mechanics, vol. XXIX (pp. 81718185). Buenos Aires, Argentina.

Argenta, M. A. 2011. Metodologia para a simulação computacional do comportamento mecânico de estruturas trabeculares ósseas específicas. Tese de doutorado, Universidade Federal do Paraná, Curitiba.

Azevedo, C. A. C. 2007. Formulação alternativa para análise de domínios não-homogêneos e inclusões anisotrópicas via MEC. Dissertação de mestrado, Universidade de São Paulo, São Carlos.

Benveniste, Y. 1987. A new approach to the application of Mori-Tanaka's theory in composite materials. Mechanics of Materials, vol. 6, 147-157.

Böhm, H. J. 2010. Continuum micromechanics of materials. Institut für Leichtbau und Struktur-Biomechanik. TU Wien.

Cavalcante, M. A. A. 2006. Modelagem do comportamento termo-mecânico transiente de estruturas de materiais compósitos pela teoria de volumes finitos. Dissertação de mestrado, Universidade Federal de Alagoas, Maceió.

Eshelby, J. D. 1957. The Determination of the elastic field of an ellipsoidal inclusion and related problems. Proceedings of the Royal Society of London, Vol. 241, (pp. 376-396). London, United Kingdom.

Eshelby, J. D. 1959. The elastic field outside an ellipsoidal inclusion. Proceedings of the Royal Society of London, Vol. 252, (pp. 561-569). London, United Kingdom.

Kawashita, M. \& Nozaki, H. 2001. Eshelby tensor of a polygonal inclusion and its special properties. Journal of Elasticity, vol. 64, 71-84.

Kurukuri, S. 2004. A review of homogenization techniques for heterogeneous materials. Short-term paper, Bauhaus Universität, Weimar.

Lai, W. M., Rubin, E. \& Krempl, D. 1993. Introduction to Continuum Mechanics. Burlington, USA: Ed. Butterworth Heinemann, $556 \mathrm{p}$.

Lorenci, G. V. S. 2013. Formulação micromecânica do comportamento poroelástico de um meio rochoso fraturado. Dissertação de mestrado, Universidade Federal do Rio Grande do Sul, Porto Alegre.

Ma, H. 2010. Solutions of Eshelby-type inclusion problems and a related homogenization method based on a simplified strain gradient elasticity theory. Master's dissertation, Texas A\&M University, College Station.

Mura, T. 1987. Micromechanics on defects in solids. Dordrecht, Netherlands: Ed. Martinus Nijhoff Publishers, 587 p.

Rodin, G, J. 1996. Eshelby's inclusion problem for polygons and polyhedra. Journal of the Mechanics and Physics of Solids, vol. 44, No. 12, 1977-1995.

Soares, G. F. P. 2010. Homogeneização de um material composto formado por uma matriz polimérica com uma segunda fase particulada. Tese de doutorado, Universidade Federal do Rio Grande do Sul, Porto Alegre.

Tavares, J. R. A. V., Silva, R. M. S. \& Silva, J. G. T. 2012. Estudo experimental e numérico do módulo de elasticidade efetivo do concreto. Anais do VII Congresso Norte Nordeste de Pesquisa e Inovação, vol. 1, disponível em: < http://propi.ifto.edu.br/ocs/index.php/connepi/vii/paper/viewFile/779/2104 >. Palmas, Brasil.

Tian, L. \& Rajapakse, R. K. N. D. 2007. Elastic field of an isotropic matrix with nanoscale elliptical inhomogeneity. International Journal of Solids and Structures, vol. 44, 7988-8005.

Weinberger, C., Cai, W., \& Barnett, D. 2005. Lecture Notes - Elasticity of Microscopic Structures. Available in: < http://micro.stanford.edu/ caiwei/me340b/content/me340b-notes_v01.pdf >, 118 p.

Xu, B. \& Wang, M. 2005. Special properties of Eshelby tensor for a regular polygonal inclusion. Acta Mechanica Sinica, vol. 21, 267-271.

Zou, W., He, Q., Huang, M. \& Zheng, Q. 2010. Eshelby's problem of non-elliptical inclusions. Journal of Mechanics and Physics of Solids, vol. 58, 346-372.

\section{RESPONSABILIDADE AUTORAL}

“Os autores são os únicos responsáveis pelo conteúdo deste trabalho”.

\section{EVALUATION OF THE INCLUSION BOUNDARY CONDITIONS APPROACH EFFECTS OF TWO-PHASE MATERIAL}

\author{
Camila Francisca de Melo, mila_f_melo@hotmail.com ${ }^{1}$ \\ Marco André Argenta, marco.argenta@gmail.com²
}

\footnotetext{
${ }^{1}$ Universidade Federal do Paraná - Programa de Pós-Graduação em Métodos Numéricos em Engenharia, Centro Politécnico da UFPR - Curitiba - Paraná/Brasil
} 
${ }^{2}$ Universidade Federal do Paraná - Programa de Pós-Graduação em Métodos Numéricos em Engenharia, Centro Politécnico da UFPR - Curitiba - Paraná/Brasil

Abstract: Considering an isotropic two-phase material (inclusion and matrix), this work aims is to analyze the mechanical behavior of body through the location of the inclusion in the matrix, beginning with a central inclusion and approaching this inclusion to the boundary conditions. This hypothesis will be tested with a homogenized structure and another one with an explicit inclusion and matrix. For this to occur, the Mori-Tanaka homogenization's method is used, in order to obtain the constitutive tensor for this isotropic two-phase solid. To calculate the effective properties of the composite, an algorithm was implemented in Python language and for the modeling and discretization of the structure, finite elements software Ansys is used. The evaluation of the proposal is summarized in a comparative analysis of the results, which showed that the results are plausible and provide some considerations about the effect of the inclusions in the boundary conditions of the matrix.

Keywords: Numerical methods, homogenization's method, inclusion, two-phase material, finite elements. 